\title{
MALADIE DE FORESTIER REVELEE PAR UNE DYSPHAGIE A PROPOS DE DEUX CAS
}

\author{
I. HARIGA, CH. MBAREK, H. OUNI, CH. CHAMMEKHI*, O. BEN GAMRA, M. BEN AMOR, \\ S. ZRIBI, A. EL KHEDIM \\ SERVICE D'ORL ET DE CHIRURGIE CERVICO-FACIALE \\ *SERVICE DE RADIOLOGIE \\ HÔPITAL HABIB THAMEUR, TUNIS
}

\begin{abstract}
La maladie de Forestier ou hyperostose ankylosante vertébrale engainante est un désordre musculo-squelettique non inflammatoire responsable d'une ossification ligamentaire essentiellement du ligament longitudinal antérieur. Elle touche de préférence l'homme de plus de 50 ans. La dysphagie est un symptôme rarement observé dans l'évolution de la maladie de Forestier.

Nous rapportons deux cas révélés par une dysphagie isolée. II s'agit de deux hommes âgés de 50 et 56 ans.

Le diagnostic était radiologique. La radiographie standard du rachis a objectivé des ostéophytes du rachis cervical. Le transit oesogastroduodénal a montré une compression modérée de l'œsophage cervical.

Le traitement était médical reposant sur les anti inflammatoires non stéroïdiens et les antalgiques. Le traitement chirurgical est indiqué dans les cas de dysphagie sévère.

Mots clés : maladie de Forestier, hyperostose vertébrale, dysphagie.
\end{abstract}

Forestier's disease now called DISH (Diffuse Idiopathic Skeletal Hyperostosis) is a non inflammatory esthesopathy ossifying the anterolateral spine and sparing the disc and joint space in elderly men.

Cervical spine involvement rarely leads to dysphagia. We report two cases of Forestier's disease revealed by this symptom. Radiology provides the diagnosis.

Surgery is indicated in case of severe dysphagia or no improvement with medical treatment.

Key words : Forestier's disease, Skeletal Hyperostosis, dysphagia.

\section{INTRODUCTION}

La maladie de Forestier ou hyperostose ankylosante sénile rachidienne est une maladie rhumatologique d'étiologie inconnue décrite pour la première fois en 1950 par J. Forestier et Rotes-Querol (1).

Elle est caractérisée par une ossification des ligaments paravertebraux et des enthèses périphériques (2).

Le plus souvent asymptomatique, elle est de découverte fortuite sur des radiographies standards. Plus rarement elle se manifeste par des douleurs cervicales, une raideur rachidienne ou une dysphagie.

Nous rapportons deux cas de maladie de Forestier révélée par une dysphagie.

\section{OBSERVATION}

\section{Observation $\mathrm{N}^{\circ} \mathrm{1}$ :}

Monsieur A. G. âgé de 50 ans sans antécédents pathologiques notables consulte pour une dysphagie haute aux solides évoluant depuis 6 mois, associée à une asthénie sans altération de l'état général ni notion de fausses routes. L'examen ORL et l'examen neurologique étaient sans particularités.

La biologie n'a pas montré de trouble métabolique ni de syndrome inflammatoire.

La Radiographie du rachis cervical de face et de profil (Fig. 1) a mis en évidence des ostéophytes cervicaux antérieurs C5-C6 et C6-C7 et une hyperostose vertébrale avec un foramen intact.

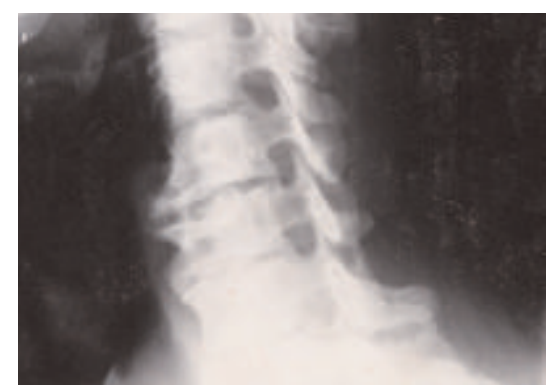

Fig. 1 : Radiographie du rachis cervical de profil, ostéophytes cervicaux antérieurs C5-C6 et C6-C7

Le transit oesogastroduodénal (TOGD) a objectivé une compression étagée de la paroi postérieure de l'œsophage (Fig.2).

La Myélographie Statique et dynamique (Fig.3) a montré une perte de la lordose cervicale. On a noté l'absence d'in- 
stabilité intervertébrale ou de sténose cervicale dynamique. Le traitement était symptomatique reposant sur des antiinflammatoires non stéroïdiens et des antalgiques.

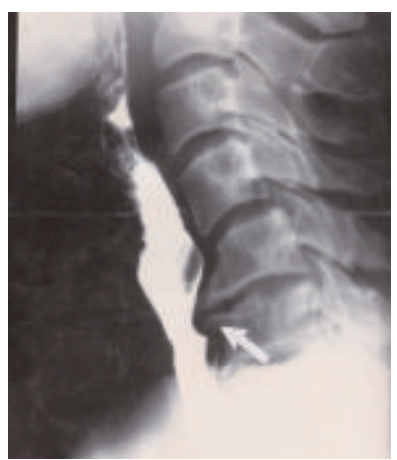

Fig. 2 : TOGD : compression étagée de la paroi postérieure de l'œsophage

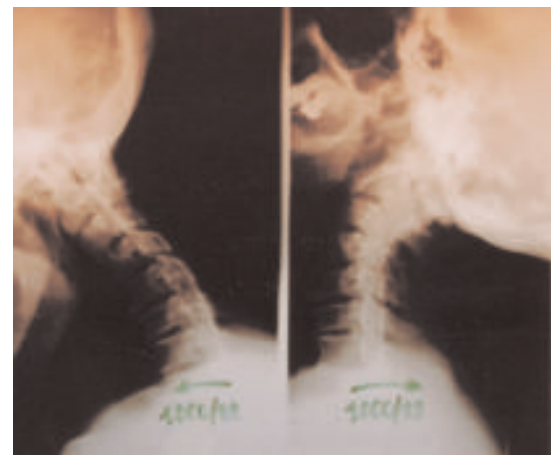

Fig. 3 : Myélographie : perte de la lordose cervicale

\section{Observation N 2 :}

Monsieur J.H âgé de 56 ans, est adressé pour dysphagie aux solides d'aggravation progressive depuis quelques mois, isolée sans dyspnée, ni altération de l'état général. Les examens cliniques et neurologiques étaient normaux. La mobilité rachidienne était conservée.

Les radiographies standards ont révélé des lésions d'arthrose cervicale étagée avec ostéophytose antérolatérale en regard de $\mathrm{C} 4, \mathrm{C} 5$ et $\mathrm{C} 6$.

Le TOGD a montré une compression de la paroi postérieure de l'œsophage en regard de l'osteophytose.

Le patient était mis sous anti-inflammatoires non stéroïdiens et des antalgiques.

\section{DISCUSSION}

La maladie de Forestier encore appelée hyperostose ankylosante vertébrale engainante ou hyperostose ankylosante sénile rachidienne, est une enthésopathie non inflammatoire responsable d'un désordre musculo-squelettique. Elle est caractérisée par une hyperostose exubérante "en flamme de bougie" des faces antérolatérales des corps vertébraux (3) avec une prédominance thoracique $(1,4,5)$. Elle affecte des patients âgés de plus de 50 ans, avec un sex ratio de 3 (6).

D'étiologie inconnue, certains mécanismes ont été évoqués tel qu'un désordre du métabolisme de la vitamine A, ou un rôle de l'insuline par stimulation de l'hormone de croissance. On incrimine l'obésité, I'hyperuricémie et le diabète de type II (7). Aucun facteur de risque n'a été noté chez nos patients.

La maladie est le plus souvent asymptomatique de découverte radiologique fortuite. Elle peut se manifester par une douleur rachidienne généralement modérée associée à une raideur. Le rachis cervical est atteint dans 14 à $76 \%$ des cas (8).

Les complications neurologiques telles que les compressions médullaires ou radiculaires, les compressions nerveuses périphériques, les paralysies récurrentielles laryngées sont rares (9).

La dysphagie est un symptôme révélateur de la maladie de Forestier dans 0,1 à $28 \%$ des cas (10). Elle est due à plusieurs mécanismes. La compression mécanique directe du pharynx et/ou de l'oesophage par l'hyperostose antérieure provoque une dysphagie par obstruction (11). C'est le cas de nos deux malades. A un stade plus avancé, les ostéophytes enchâssés dans la paroi musculaire peuvent induire des troubles fonctionnels du péristaltisme qui vont s'aggraver ensuite par l'inflammation et par la réaction de fibrose musculaire, réalisant ainsi une forme pseudo-tumorale (12).

La dysphagie est en général plus marquée pour les solides que pour les liquides, s'améliore par la flexion du rachis cervical et s'aggrave par l'extension (11). Elle peut s'accompagner d'odynophagie, de stase salivaire, de dysphonie, de dyspnée, d'apnée du sommeil ou de fausses routes $(9,11)$.

Le diagnostic est évoqué sur les radiographies standards du rachis. La tomodensitométrie (TDM) permet d'apprécier l'extension de l'ossification en forme de "double corps vertébral" vers les organes de voisinage ainsi que le calibre du canal rachidien et des foramens inter-vertébraux (13).

L'imagerie par résonance magnétique (IRM) est indiquée en cas de complications neurologiques.

Le transit oesophagien est demandé dans le cadre de l'exploration d'une dysphagie (3).

Le diagnostic de la maladie de Forestier se base sur des critères radiologiques établis par Resnick et al. (8) dans les années 70 et qui sont toujours d'actualité :

1. Calcifications et ossifications le long de la face antérolatérale de 4 corps vertébraux contigus.

2. Relative préservation de la hauteur des disques intervertebraux dans les zones atteintes.

3. Absence d'ankylose des articulations inter-apophysaires postérieures et absence de sclérose des articulations sacro-iliaques.

L'endoscopie oesophagienne, au mieux avec un endoscope souple permet d'évaluer la compression et d'apprécier 
l'état trophique de la muqueuse pharyngo-oesophagienne (3).

Le diagnostic différentiel se pose avec l'arthrose et les spondylopathies. C'est là que les critères de Resnick prennent toute leur importance.

II n'existe pas à nos jours de traitement préventif de la maladie de Forestier. La physiothérapie peut améliorer la raideur rachidienne. Le traitement médical repose sur les anti-inflammatoires non stéroïdiens, voire des bolus de corticoïdes associés à un myorelaxant et à un régime antireflux.

Le traitement chirurgical est indiqué dans les cas de dysphagie sévère ou hyperostose cervicale très proéminente devant le risque de fausses routes (14).

\section{CONCLUSION}

La maladie de Forestier se manifeste par une dysphagie. Ce symptôme ne doit pas être négligé car il expose à des fausses routes et à des lésions irréversibles de la paroi postérieure de l'oropharynx.

Le diagnostic est essentiellement radiologique. La prise en charge de la maladie ne sera entreprise qu'après avoir éliminé une pathologie tumorale pharyngo- oesophagienne. Le recours à la chirurgie est justifié dans les formes sévères.

\section{REFERENCES}

1- Forestier J., Rotes-Querol J. Senile ankylosing hyperostosis of the spine. Ann Rheum 1950 1950; 9:321-30.

2- Taillandier J. Hyperostose vertébrale ankylosante. Revue de rhumatisme 2004; 71; 6: 525-26.

3- Riffaud L., Mohr E., Morandi X. Maladie de Forestier revelée par une dysphagie. Presse Med 2002; 31: 1650-2.

4- Mazieres B, Rovensky J. Non inflammatory enthesopathies of the spine . A diagnostic approach. Bailliers Best Pract Res Clin Rheumatol 2000; 14: 201-17. 5 - Minco JF, Person M, Dam Mien P. Dysphagie et éperon osseux cervicaux. Neurochirurgie 2001; 47: 140-2.

6- Dioré K., Facon D., Marsot-Dupuch K. Une cause de dysphagie méconnue: la maladie de Forestier. J Fr ORL 2005; 88: 124-7.

7- Littlejohn Go, Mall S. Difflux idiopathic skeletal hyperostosis and new bone formation in male gouty subjects. Rheumatol Int 1982; 2: 83-6.

8- Resnick D, Niwayama G. Radiographic and pathologic features of spinal involment in diffuse idiopathic skeletal hyperostosis (DISM). Radiology 1976; 119: 559-68.
9-Aydin E, Akodogan V, Akkuzu B. Six cases of Forestier syndrome, a rare case of dysphagia. Acta Otolaryngol $2006 ; 126(7): 775-8$.

10- Anthony J. Matan, Jack Msu, Brace A. Fredrickson. Management of respiratory compromise caused by cervical osteophytes: a case report and review of the literature spine Journal 2002; 2: 456-9.

11- Ebo D, Goethals L, Bracke P. Dysphagia in a patient with giant osteophytes: case presentation and review of the literature. Clin Rheumatol 2000; 19: 70-2.

12- Squadrito G, Reale F, D’Agostino G. Combined diagnostic imaging in a patient with Forestier disease and dysphagia. Rays 2005; 30(3): 251-5.

13- Commisa M, De Serio A, Guglielmi G. Diffuse idiopathic skeletal hyperostosis. Eur J Radiol 1998; 27: S7-S11.

14- Matgé G. Radiculopathie cervicale au cours de la maladie de Forestier : Traitement chirurgical. A propos d'un cas et revue de la littérature. Neurochir. 2005; $51: 15-18$ 